\title{
Supporting Information Automated Analysis of Orientational Order in Images of Fibrillar Materials
}

Nils E. Persson ${ }^{\dagger}$, Michael A. McBride ${ }^{\dagger}$, Martha A. Grover ${ }^{\dagger *}$,Elsa Reichmanis ${ }^{\dagger+*}$

${ }^{\dagger}$ School of Chemical \& Biomolecular Engineering, Georgia Institute of Technology, 311 Ferst Dr, Atlanta, GA 30332.

${ }^{*}$ School of Chemistry \& Biochemistry, Georgia Institute of Technology, 901 Atlantic Drive Atlanta, GA 30332.

${ }^{\ominus}$ School of Materials Science and Engineering, Georgia Institute of Technology, 771 Ferst Dr. NW, Atlanta, GA 30332.

*Authors to whom correspondence should be addressed: martha.grover@chbe.gatech.edu, elsa.reichmanis@chbe.gatech.edu 


\section{S1. The Structure Tensor}

The structure tensor maps angles and vectors into a space in which linear averaging is valid, and thus filtering techniques such as Gaussian filtering can be effective. Consider, for example, the set: $\left\{89^{\circ}, 90,91,269,271,180\right\}$. These angles all point more or less vertically, except for $180^{\circ}$, which is horizontal. Their numerical average is $165^{\circ}$ and their median is $135.5^{\circ}$. These values are clearly not meaningful statistics for this set of angles - the average and median are both significantly horizontally biased. Statistics for angles are much more rationally computed in terms of the structure tensor, defined as: ${ }^{1}$

$$
J=\left[\begin{array}{cc}
\cos (\theta)^{2} & \cos (\theta) \sin (\theta) \\
\cos (\theta) \sin (\theta) & \sin (\theta)^{2}
\end{array}\right]=\left[\begin{array}{ll}
J_{x x} & J_{x y} \\
J_{x y} & J_{y y}
\end{array}\right]
$$

For two-component vectors, this is the same as taking the outer product. For the above set of angles, we end up with the following set of structure tensors (omitting the $3^{\text {rd }}$ entry because it is identical to the $2^{\text {nd }}$ ):

Table S1. Demonstration of the averaging of orientations via the structure tensor.

\begin{tabular}{|l|lll|}
\hline Angle & $\boldsymbol{J}_{\mathbf{x x}}$ & $\boldsymbol{J}_{\mathbf{x y}}$ & $\boldsymbol{J}_{\mathbf{y y}}$ \\
\hline $\mathbf{8 9}^{\circ}$ & 0.0003 & 0.0174 & 0.999695 \\
$\mathbf{9 0}$ & 0 & 0 & 1 \\
$\mathbf{9 1}$ & 0.0003 & -0.0174 & 0.999695 \\
$\mathbf{2 6 9}$ & 0.0003 & 0.0174 & 0.999695 \\
$\mathbf{2 7 1}$ & 0.0003 & -0.0174 & 0.999695 \\
$\mathbf{1 8 0}$ & 1 & 0 & 0 \\
Average & 0.1669 & 0 & 0.8331 \\
\hline
\end{tabular}

$J_{\mathrm{xx}}$ describes the overall x-component of an orientation, i.e. "how horizontal", $J_{\mathrm{yy}}$ describes the overall vertical component of an angle, and $J_{\mathrm{xy}}$ describes "how positively diagonal." An angle of $45^{\circ}$ would have the highest possible value of $J_{\mathrm{xy}}$, whereas an angle of $-45^{\circ}$ would be the lowest.

From the averages of each element of the $J$ matrices, we re-form a matrix $J_{\text {average, }}$ and take its eigenvalues and eigenvectors:

$$
\operatorname{eig}\left(J_{\text {average }}\right)=\operatorname{eig}\left(\left[\begin{array}{cc}
0.1669 & 0 \\
0 & 0.8331
\end{array}\right]\right)=\left[\begin{array}{ll}
1 & 0 \\
0 & 1
\end{array}\right],\left[\begin{array}{cc}
0.1669 & 0 \\
0 & 0.8331
\end{array}\right]
$$

The eigenvector with the highest eigenvalue $(0.8331)$ indicates the orientation of $J_{\text {average }}$, so [0; 1$]$ is the average, a straight vertical vector, which makes perfect sense in the context of our hypothetical set. The above procedure is applied to find the black lines that are plotted at the average of each orientation distribution. When using the structure tensor for diffusion filtering, the scheme illustrated in Figure S1 applies. 


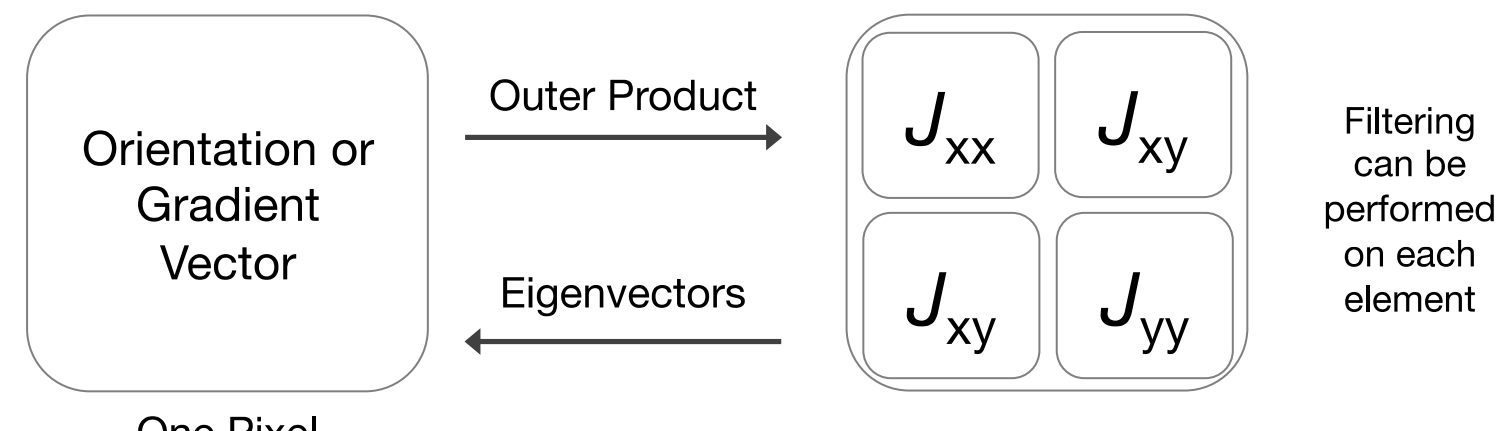

Figure S1. Diagram of the use of the structure tensor. An $m \times n$ image is converted into an $m \times n$ $\times 4$ image, where each pixel has a corresponding $J_{\mathrm{xx}}, J_{\mathrm{xy}}$, and $J_{\mathrm{yy}}\left(J_{\mathrm{xy}}\right.$ is repeated and thus redundant). Gaussian filtering can be applied to each layer of the $m \times n \times 4$ image, and then each pixel's structure tensor is reformed, eigenvectors are computed, and a filtered orientation is returned.

\section{S2. Effect of Filtering Parameters on Image Analysis Results}

\section{S2.1. Effect of Orientation Smoothing}

Each element of the structure tensor is smoothed by an isotropic Gaussian filter of standard deviation $\rho$ prior to making each diffusion time step. Local image gradient vectors have significant variance, even at fiber edges. This parameter smoothens the direction of the diffusion tensor (which is computed directly from the structure tensor) so that pixel intensities diffuse along the average fiber orientation in a given locality. Heuristically, the value of this parameter should not exceed the typical fiber width, otherwise orientations from high-intensity fibers will influence that of their neighbors. The impact of this parameter is less visible in the gray scale filtering result than it is in the final orientation map, so examples are provided to illustrate its impact below. It is clear that this parameter should be held constant when comparing results across multiple images. 
Orientation

Smoothing

Sigma
CED Filter

Result
Orientation Map
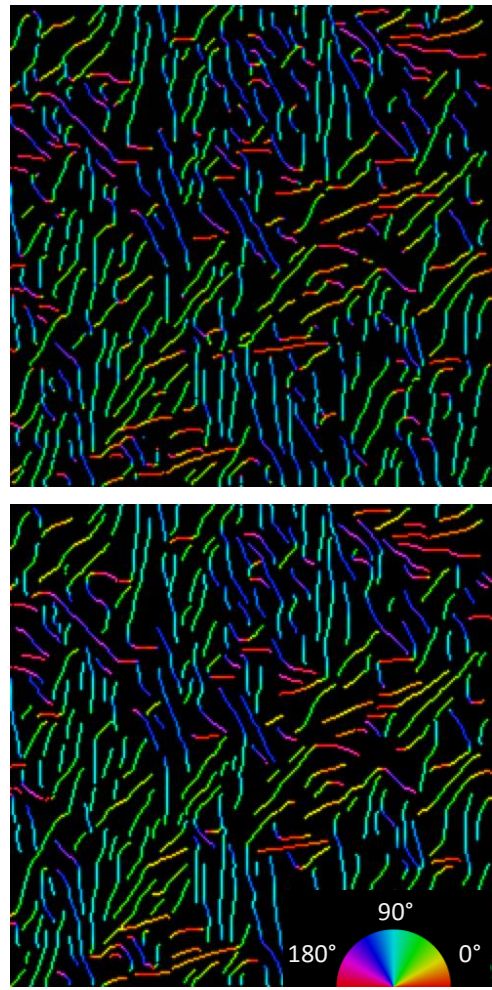

$40 \mathrm{~nm}$
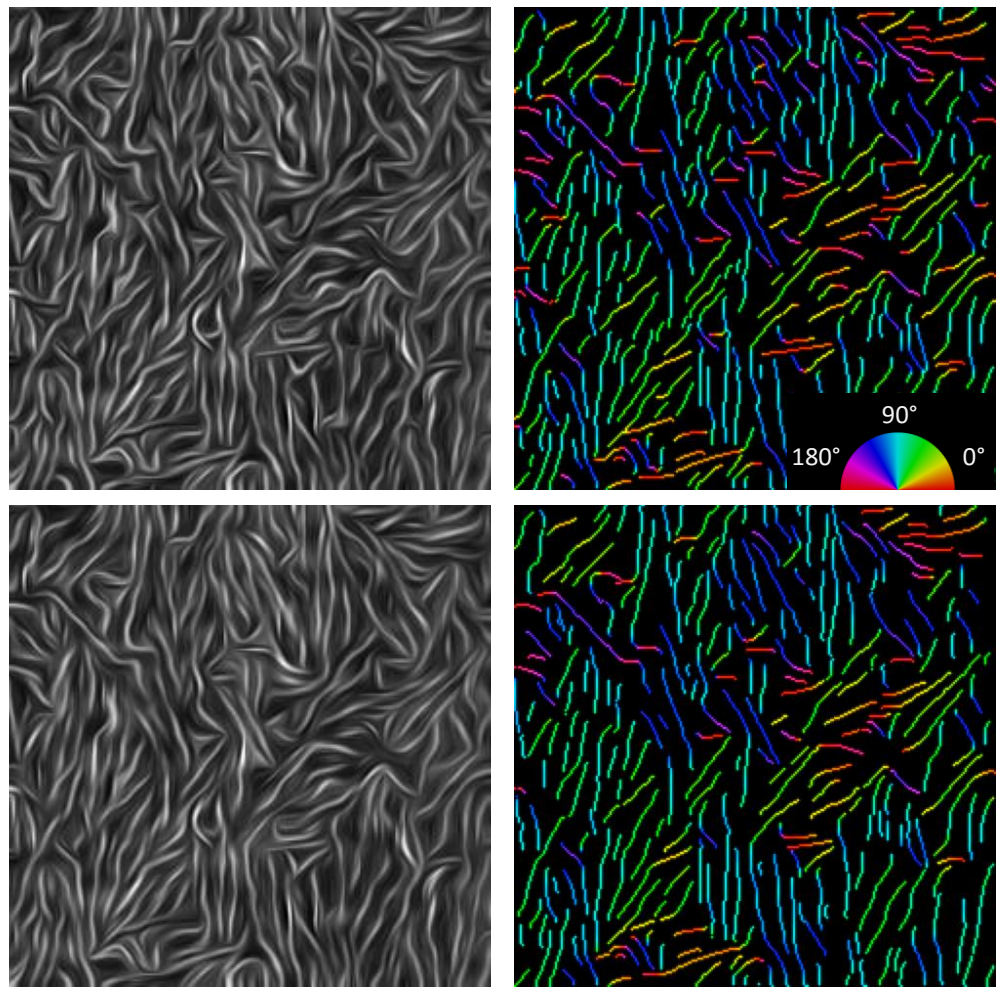
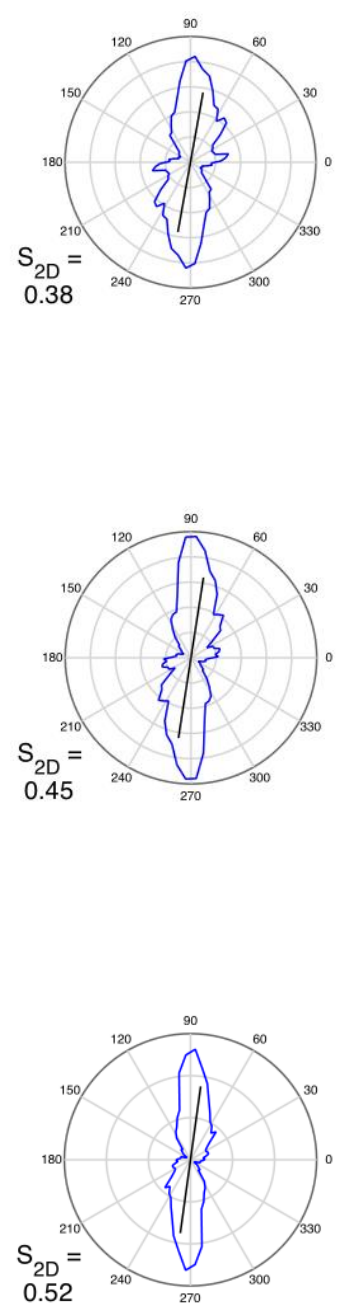

Figure S2. The effect of structure tensor smoothing. Standard deviation of orientation in $\mathrm{nm}$ indicated at left, followed by the gray scale result of diffusion filtering, the Orientation Map, and an orientation distrubtion. Other parameters used for this filter: initial Gaussian smoothing, $10 \mathrm{~nm}$; diffusion time, $8 \mathrm{~s}$; top hat size, 30nm; adaptive thresholding; noise removal area, 3500 $\mathrm{nm}^{2}$; skeleton fringe removal, 40nm. Original image is shown at the left of Figure S3.

\section{S2.2. Effect of Diffusion Time}



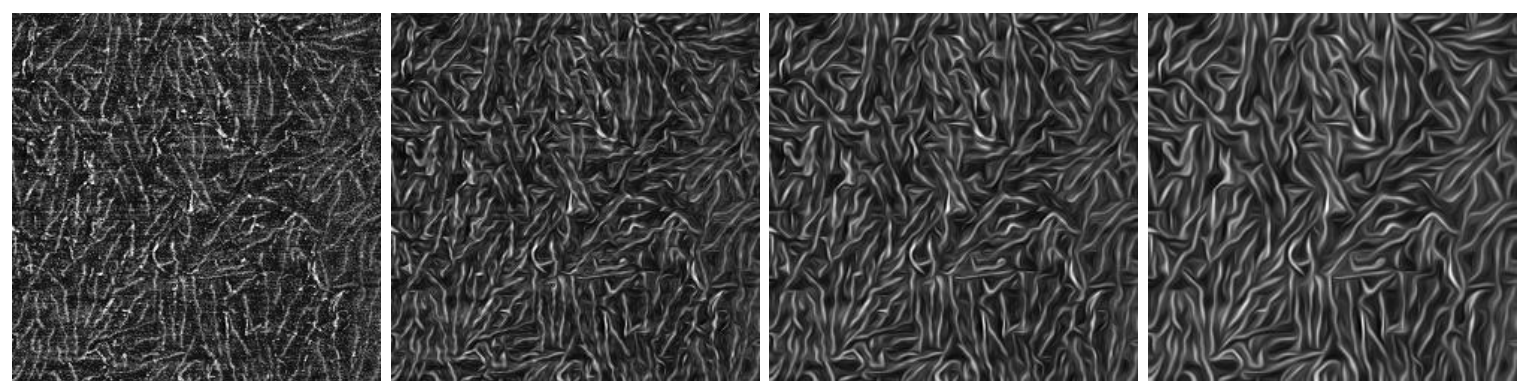

Figure S3. The effect of the "diffusion time" parameter on diffusion filtering results, from left to right: original gray scale, $1 \mathrm{~s}$ diffusion time, $3 \mathrm{~s}$, 8s. Constant parameter values: initial Gaussian smoothing, $10 \mathrm{~nm}$; orientation smoothing, $30 \mathrm{~nm}$.

Gray values diffuse by a default time step of 0.15 s until a time specified by the user in the field "diffusion time". The effect of time step is negligible, as long as it is fractions of a second. The only consideration for choosing a different time step would be for computational efficiency. However, as diffusion is allowed to proceed, one observes a pronounced smoothing of fibrillar regions. Wrinkles and kinks in fibers tend to be straightened, and segments that may have appeared to be separate may connect. CED filtering preserves the average intensity of the image, so no wash-out will occur. While the $8 \mathrm{~s}$ diffusion time appears over-smoothed in the gray image, it yields a more accurate skeleton than shorter diffusion times. The orientation distribution and $S_{2 \mathrm{D}}$ are not strongly affected by diffusion time, so it is more of an aesthetic choice. The user is encouraged to explore this parameter through the GUI to determine what works best for their application.

\section{S2.3. Effect of Top Hat Filter Size}
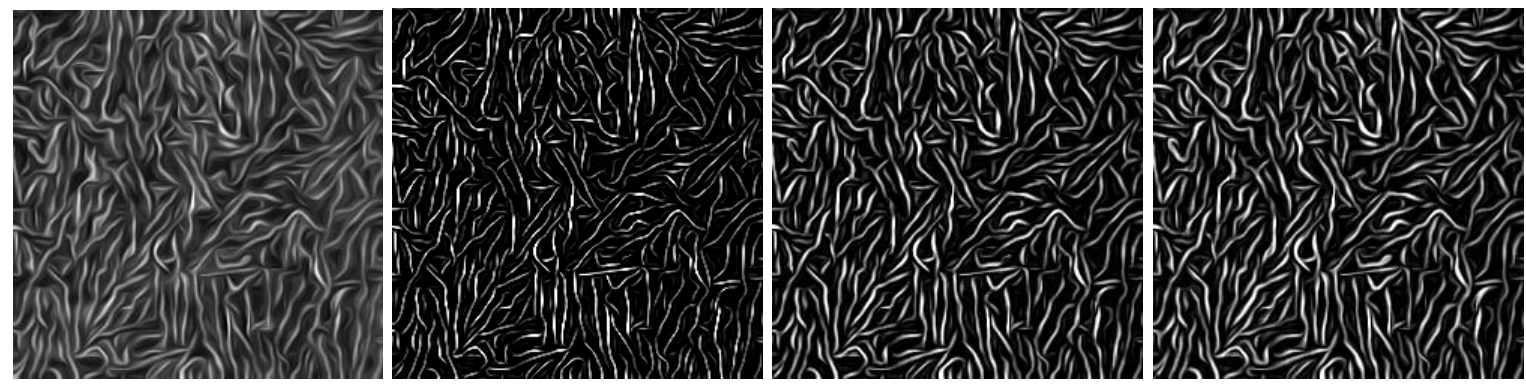

Figure S4. The effect of the "top hat size" parameter on contrast enhancement, from left to right: initial result of diffusion filtering, result of $10 \mathrm{~nm}$ top hat filter, $30 \mathrm{~nm}$, 50nm. Constant parameter values: initial Gaussian smoothing, 10nm; orientation smoothing, 30nm; diffusion time, 8s.

The top hat filter is a robust contrast-enhancing filter that is incredibly effective after diffusion filtering has been applied. It enhances sharp peaks in an image and suppresses the background. The images in Figure $\mathbf{S 4}$ are all gray scale - still no thresholding has been applied. The top hat size parameter determines the radius of the disk-shaped structuring element used for the erosion and dilation operations performed during top hat filtering. It is apparent from these images that a top hat size greater than or equal to the smallest fiber width should be used; very small top hat size excessively erode the thinnest, lightest fibers. It should be noted that the actual top hat disk radius used in the program is converted from nm to pixels and rounded up to the nearest pixel size - only integers are allowed for this morphological operation. 

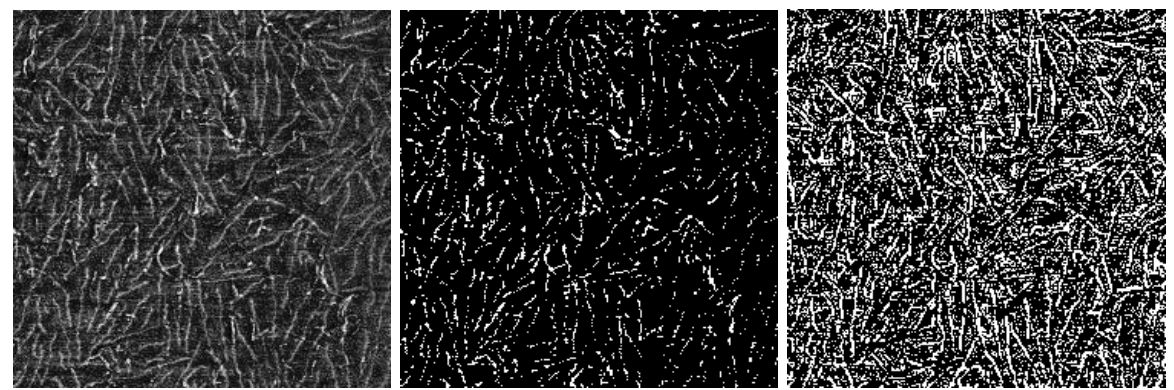

Figure S5. Thresholding of original image. Left: original gray scale. Center: Automatic global threshold. Right: Adaptive threshold surface using Yanowitz-Bruckstein method. ${ }^{2}$

Figure S5 shows traditional thresholding approaches applied to the original gray scale image. Clearly the smoothing and contrast enhancing filters were necessary before thresholding. Two thresholding options are offered to convert the result of top hat filtering into a binary image: adaptive thresholding using the method introduced by Yanowitz and Bruckstein, ${ }^{2}$ and a simple global threshold. Noise removal is also offered to clean the resulting binarized image: connected components (contiguous white regions) of area less than the specified maximum noise area are removed. The effect of these operations are shown in Figures S6 and S7.
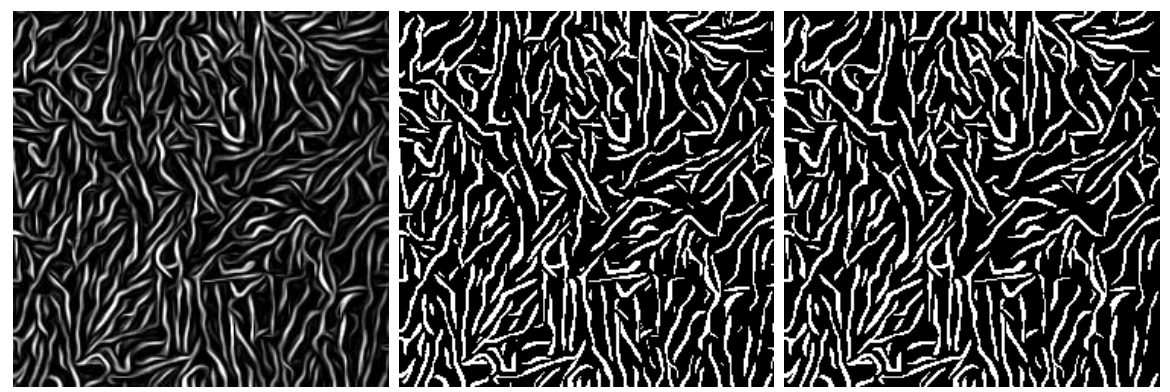

Figure S6. Thresholding of image after diffusion and top hat filtering. Left: gray scale top hat filter result. Center: global threshold at gray value of 0.3. Right: Noise removed if area $<3500$ $\mathrm{nm}^{2}$.
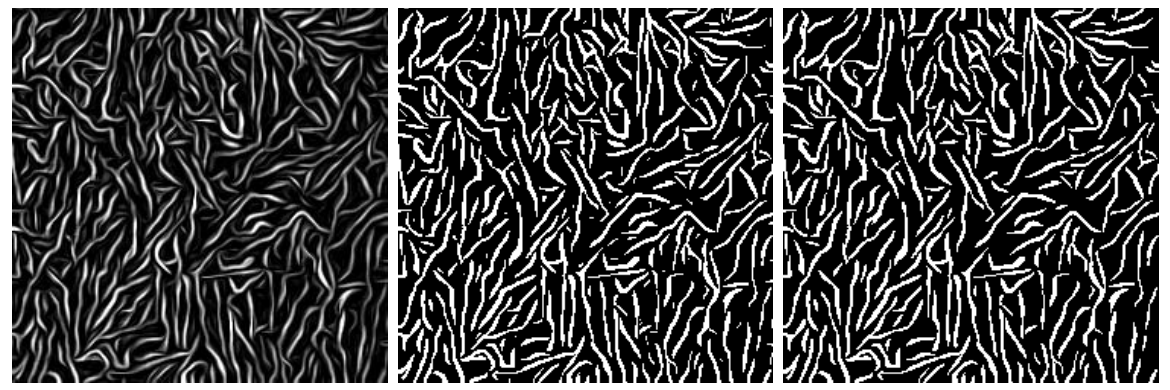

Figure S7. Thresholding of image after diffusion and top hat filtering. Left: gray scale top hat filter result. Center: result of adaptive thresholding. Right: Noise removed if area $<3500 \mathrm{~nm}^{2}$.

Since the contrast-enhanced diffusion and top hat filtered image is already so clear, thresholding is now trivially simple. Even a global threshold of 0.3 yields nearly the same result as a more 
advanced adaptive thresholding operation. Additionally, the binarized structure is very smooth, which aids in the skeletonization process.

\section{S2.5. Skeletonization}
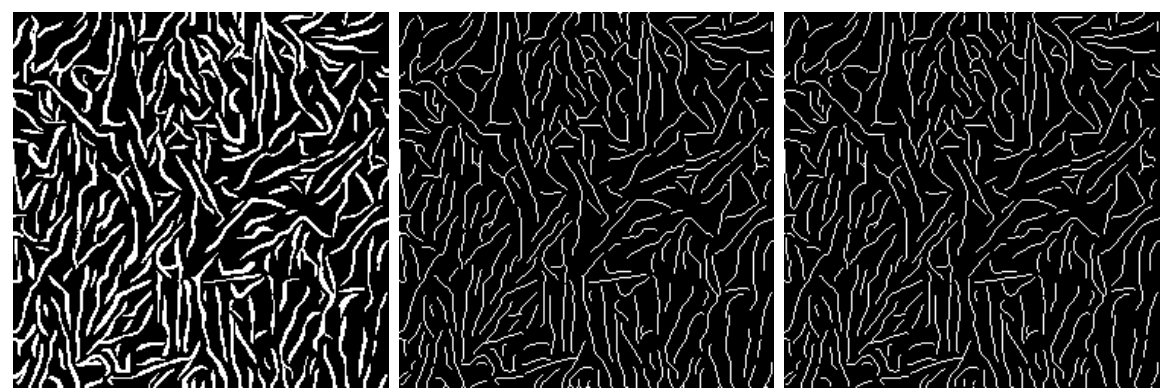

Figure S8. Skeletonization and trimming. Left: result of adaptive thresholding and noise removal. Center: result of skeletonization. Right: skeleton after removal of branches less than $40 \mathrm{~nm}$ long.

Skeletonization is the final processing step to be performed. It is a common morphological operation with a built-in MATLAB function that thins connected white components to single-pixel width. Bumps along the edges of the white components can cause the skeleton to produce small fringe branches along the main backbone, so a feature has been included that removes fringe branches of a specified length or less. Very few fringe branches can be seen in Figure S8, so a better example is provided in Figure S9. Essentially, this algorithm removes pixels if the sum of their geodesic distances from their nearest branch point and end point is less than the specified length, and that pixel lies between its nearest branch and end points (indicating that it is actually on a branch).
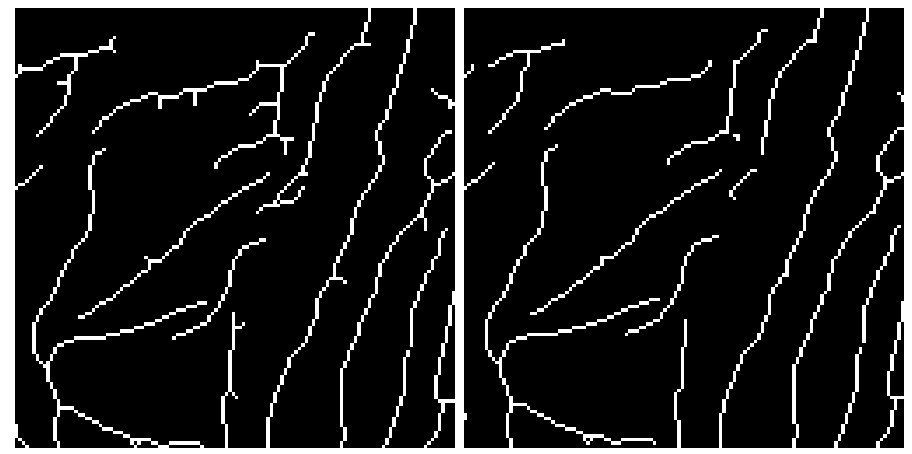

Figure S9. Skeleton fringe trimming, before and after.

\section{S3. Anisotropic Diffusion Filtering}

Anisotropic diffusion filtering applies the principles of two-dimensional Fickian diffusion to the enhancement of images. Gray levels are the species undergoing diffusion, where "concentrations" can range from 0 (black) to 1 (white). The average gray level of the image is maintained, adherent to the equivalent of a mass balance in particulate diffusion. Isotropic diffusion would simply spread gray levels from bright regions to dark regions until a homogeneous gray level is obtained over the entire image - this is effectively the same thing as a 
recursively applied Gaussian filter. Anisotropic diffusion implies that the diffusivity is represented as a tensor, rather than a scalar, at each pixel.

The $\mathrm{m} \times \mathrm{n}$ image $u$ is modeled as gray values between 0 and 1 as a function of twodimensional position and time:

$$
u(\boldsymbol{x}, t), \mathbf{x} \in\left[\left[\begin{array}{l}
0 \\
0
\end{array}\right],\left[\begin{array}{c}
m \\
n
\end{array}\right]\right]
$$

The diffusion equation to be integrated is the following, with a no-flux boundary condition at the image edges:

$$
\begin{gathered}
\frac{\partial u}{\partial t}=\operatorname{div}(\boldsymbol{D} \nabla u), t \in(0, \infty) \\
-\boldsymbol{D} \nabla u=0, x=0, m ; y=0, n
\end{gathered}
$$

where $\nabla u$ is the gradient of the image and $\boldsymbol{D}$ is a $2 \times 2$ positive definite diffusion tensor.

We would like $\boldsymbol{D}$ to strengthen diffusion along the fiber directions, so that gray levels diffuse along fiber backbones, but not perpendicular to them. Furthermore, we would like regions of the image with high directional confidence to diffuse more strongly. These directions and their confidences can be obtained from the structure tensor $\boldsymbol{J}$ described above in Section S1, by taking the outer product of the image gradient:

$$
J_{0}(\nabla u)=\nabla u \nabla u^{T}
$$

A fiber can be visualized as a sharp ridge in the context of this analysis. The image gradient will be strongest at fibers' edges, and will be pointed perpendicular to fibers' backbone orientations. However, at the backbone of each fiber the image gradient will have a very low magnitude, and thus a weakly oriented structure tensor. To account for this, and to smooth noise in the locally calculated gradients, each element of $\boldsymbol{J}_{\mathbf{0}}$ is convoluted with a Gaussian filter, $K_{\rho}$ :

$$
J_{\rho}(\nabla u)=K_{\rho} *\left(\nabla u \nabla u^{T}\right)
$$

where $\rho$ is the standard deviation of the Gaussian filter. Due to the unique properties of the structure tensor described above, this accomplishes a local averaging of orientations. The more strongly oriented pixels will influence their neighbors; so the center of a fiber between two sharp edges will adopt a strong orientation pursuant to that of the edges.

Now the diffusion tensor can be constructed. It carries the same eigenvectors as $\boldsymbol{J}_{\boldsymbol{\rho}}$, but its eigenvalues are replaced with values that enhance diffusion along the coherence orientation, which is generally perpendicular to the image gradient. The eigenvalues of $\boldsymbol{J}_{\boldsymbol{\rho}}$ are sorted so that:

$$
\lambda_{1} \geq \lambda_{2}
$$

and the eigenvector $\boldsymbol{v}_{1}$ therefore corresponds to the locally averaged gradient vector, and $\boldsymbol{v}_{2}$ corresponds with the desired coherence orientation. Coherence is a measure of the anisotropy of the structure tensor, calculated as: 


$$
\kappa:=\left(\lambda_{1}-\lambda_{2}\right)^{2}
$$

The coherence is used to formulate new eigenvalues for the diffusion tensor:

$$
\begin{gathered}
\lambda_{D 1}:=\alpha \\
\lambda_{D 2}:=\left\{\begin{array}{r}
\alpha, \kappa=0 \\
\alpha+(1-\alpha) \exp (-C / \kappa), \quad \kappa \neq 0
\end{array}\right.
\end{gathered}
$$

In both of these equations, $\alpha \in(0,1)$ is a small positive parameter that ensures finite isotropic diffusion even when no coherent structures are present. An $\alpha$ of 1 results in perfectly isotropic diffusion, equivalent to a sequence of Gaussian filters. The anisotropic nature of the diffusion tensor comes from the second term of $\lambda_{D 2}$. The parameter $C$ acts as a threshold for $\kappa$ above which the diffusion tensor will become strongly anisotropic, and below which diffusion will tend toward the standard isotropic case.

To summarize, large local anisotropy in an image leads to a structure tensor with strongly differing eigenvalues, which in turn lead to a diffusion tensor with strongly differing eigenvalues. The only difference between the two tensors is that the structure tensor's largest eigenvalue corresponds to the gradient direction, while the diffusion tensor's largest eigenvalue corresponds to the direction perpendicular to the gradient, or coherence orientation. To mathematically construct the diffusion tensor $\boldsymbol{D}$, we formulate a new matrix using the eigenvectors of $\boldsymbol{J}_{\boldsymbol{\rho}}$ and the new eigenvalues calculated in Eqns. 10 and 11:

$$
\boldsymbol{D}=\boldsymbol{V}\left[\begin{array}{cc}
\lambda_{D 1} & 0 \\
0 & \lambda_{D 2}
\end{array}\right] \boldsymbol{V}^{T}
$$

where $\boldsymbol{V}$ is the matrix containing the original eigenvectors of $\boldsymbol{J}_{\boldsymbol{\rho}}$. Finally, the gray scale image is updated according to Eqns. 4 and 5 using an explicit Euler integration scheme and rotationally invariant kernels for spatial derivates. ${ }^{3}$ The default values of $\alpha, C$, and the integration time step are $0.001,1 \times 10^{-10}$, and 0.15 , respectively. The results of filtering are invariant to large deviations in these parameters, so they are not explored in depth here.

\section{S4. Top Hat Filtering}

Top hat filtering enhances sharp details and flattens the background in images. Its name is derived from the shape of its structuring element, similar to that of a top hat. The transformation consists of three steps: an erosion, a dilation, and a subtraction. The combination of erosion and dilation is called an image opening, so the top hat filter, in essence, subtracts from the original image the result of its opening. This is illustrated in Figure S10, using a structuring element of a disk of radius two pixels. 

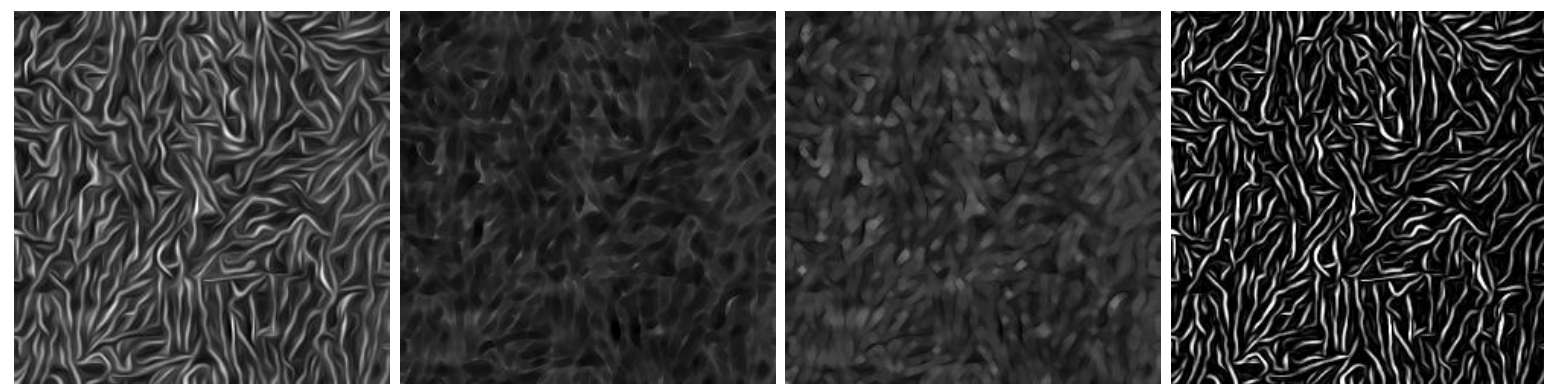

Figure S10. The top hat filtering process, from left to right: result of anisotropic diffusion filtering, result of image erosion, result of image dilation, and result of subtracting the dilated image from the result of anisotropic diffusion filtering. 

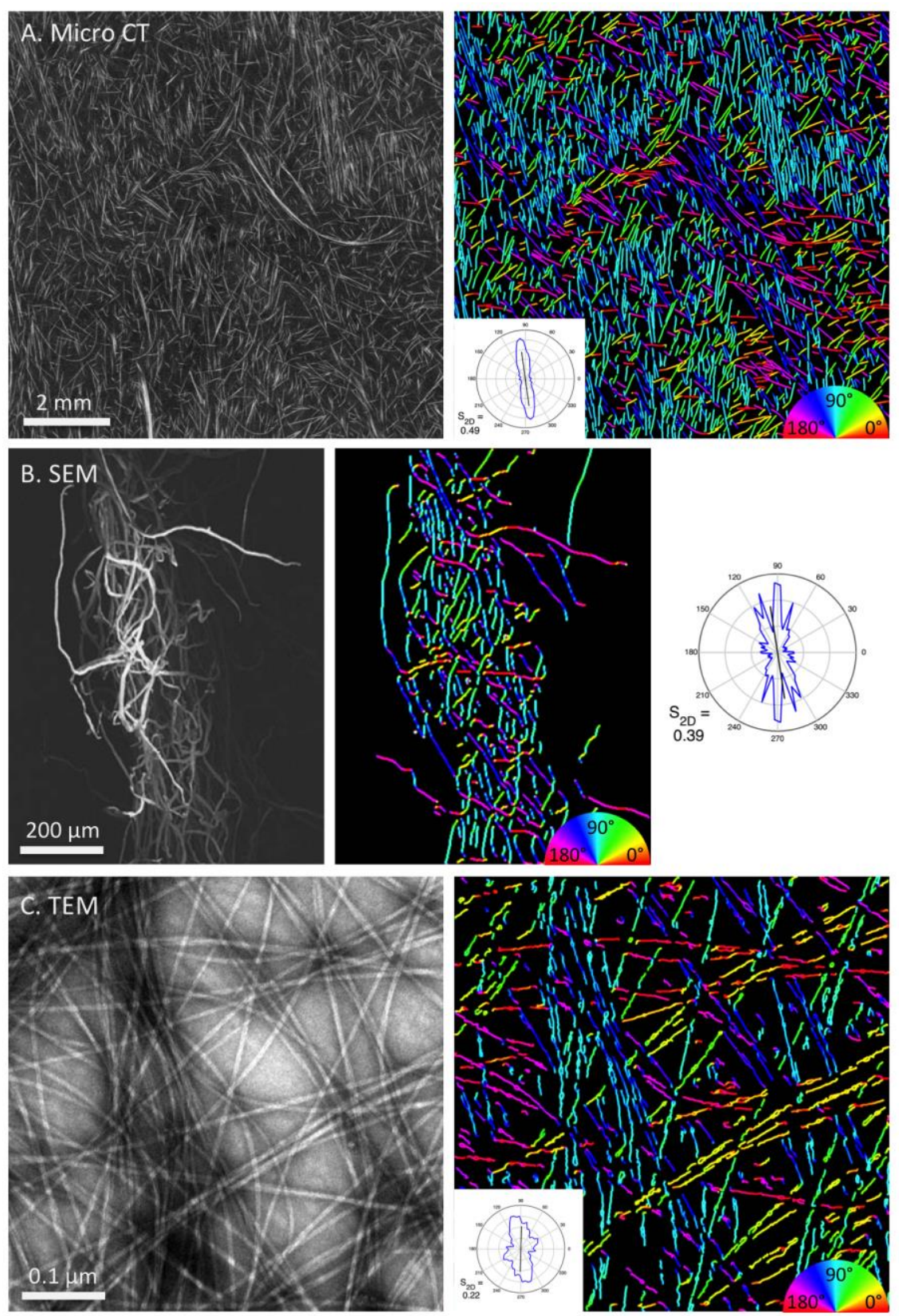

Figure S11. GTFiber applied to: (A) Micro-CT imaging (top) of a fiber composite, (B) SEM imaging of cellulose fibrils (reproduced from Ref. 4 with permission from the Royal Society of Chemistry), ${ }^{4}(\mathbf{C})$ TEM imaging of fibrillar protein assemblies.

As long as an image contains fibers whose brightness contrasts substantially with the image background, contours can be extracted and analyzed, as demonstrated in Figure S11. With different imaging systems, however, length scales can vary drastically. For the $10 \mathrm{~mm}$ micro-CT image in Figure S11A, it is impractical to enter the image size in nanometers. In this case, simply enter $5000 \mathrm{~nm}$ as the image width, and use the default filter parameters that are pre-filled in the app window. The default parameters are optimized for a $5000 \mathrm{~nm}$ image and will generally 
yield a good first result. The results in Figures S11 A-C were obtained using this strategy. In general, the units of the filtering parameters should be determined relative to the specified image size.

\section{S6. Downloading and Installing GTFiber}

The software and source code is maintained in a public GitHub repository at the following addresses:

For Mac: https://github.com/Imperssonator/GTFiber-Mac

For PC: https://github.com/Imperssonator/GTFiber-Windows

Scroll down on these pages to read the instructions on how to install the app. There are essentially three steps: download the repository, unzip it on your machine, and run "Install the App". A README file is provided explaining how to use the software once it is installed.

\section{References}

1. Weickert, J. Coherence-Enhancing Diffusion Filtering. Int. J. Comput. Vis. 1999, 31, 111127.

2. Yanowitz, S. D.; Bruckstein, a. M. A New Method for Image Segmentation. Comput. Vision, Graph. Image Process. 1989, 46, 82-95.

3. Weickert, J.; Scharr, H. A Scheme for Coherence-Enhancing Diffusion Filtering with Optimized Rotation Invariance. J. Vis. Commun. Image Represent. 2002, 13, 103-118.

4. Sharma, S.; Nair, S. S.; Zhang, Z.; Ragauskas, A. J.; Deng, Y. Characterization of Micro Fibrillation Process of Cellulose and Mercerized Cellulose Pulp. RSC Adv. 2015, 5, 63111-63122. 